\title{
A REVERSE SHOCK IN GRB 160509A
}

\author{
Tanmoy Laskar ${ }^{1,2}$, Kate D. Alexander ${ }^{3}$, Edo Berger ${ }^{3}$, Wen-fai Fong ${ }^{4,13}$, Raffaella Margutti ${ }^{5,6}$, Isaac Shivvers ${ }^{2}$, \\ Peter K. G. Williams ${ }^{3}$, Drejc Kopač ${ }^{7}$, Shiho Kobayashi ${ }^{8}$, Carole Mundell ${ }^{9}$, Andreja Gomboc ${ }^{10}$, WeiKang Zheng ${ }^{2}$, \\ Karl M. Menten $^{11}$, Melissa L. Graham ${ }^{2,12}$, and Alexei V. Filippenko ${ }^{2}$ \\ ${ }^{1}$ National Radio Astronomy Observatory, 520 Edgemont Road, Charlottesville, VA 22903, USA \\ ${ }^{2}$ Department of Astronomy, University of California, 501 Campbell Hall, Berkeley, CA 94720-3411, USA \\ ${ }^{3}$ Department of Astronomy, Harvard University, 60 Garden Street, Cambridge, MA 02138, USA \\ ${ }^{4}$ Steward Observatory, University of Arizona, 933 N. Cherry Ave, Tucson, AZ 85721, USA \\ ${ }^{5}$ Center for Interdisciplinary Exploration and Research in Astrophysics (CIERA) and Department of Physics and Astrophysics, \\ Northwestern University, Evanston, IL 60208, USA \\ ${ }^{6}$ Center for Cosmology and Particle Physics, New York University, 4 Washington Place, New York, NY 10003, USA \\ ${ }^{7}$ Faculty of Mathematics and Physics, University of Ljubljana, Jadranska 19, 1000 Ljubljana, Slovenia \\ ${ }^{8}$ Astrophysics Research Institute, Liverpool John Moores University, IC2, Liverpool Science Park, 146 Brownlow Hill, Liverpool L3 5RF, UK \\ ${ }^{9}$ Department of Physics, University of Bath, Claverton Down, Bath, BA2 7AY, UK \\ ${ }^{10}$ University of Nova Gorica, Vipavska 13, 5000 Nova Gorica, Slovenia \\ ${ }^{11}$ Max-Planck-Institut für Radioastronomie, Auf dem Huegel 69, D-53121 Bonn, Germany \\ ${ }^{12}$ Department of Astronomy, University of Washington, Box 351580 U.W., Seattle WA 98195-1580, USA \\ Received 2016 June 28; revised 2016 August 15; accepted 2016 August 29; published 2016 December 8
}

\begin{abstract}
We present the second multi-frequency radio detection of a reverse shock in a $\gamma$-ray burst. By combining our extensive radio observations of the Fermi-Large Area Telescope $\gamma$-ray burst $160509 \mathrm{~A}$ at $z=1.17$ up to 20 days after the burst with Swift X-ray observations and ground-based optical and near-infrared data, we show that the afterglow emission comprises distinct reverse shock and forward shock contributions: the reverse shock emission dominates in the radio band at $\lesssim 10$ days, while the forward shock emission dominates in the X-ray, optical, and near-infrared bands. Through multi-wavelength modeling, we determine a circumburst density of $n_{0} \approx 10^{-3} \mathrm{~cm}^{-3}$, supporting our previous suggestion that a low-density circumburst environment is conducive to the production of long-lasting reverse shock radiation in the radio band. We infer the presence of a large excess X-ray absorption column, $N_{\mathrm{H}} \approx 1.5 \times 10^{22} \mathrm{~cm}^{-2}$, and a high rest-frame optical extinction, $A_{\mathrm{V}} \approx 3.4 \mathrm{mag}$. We identify a jet break in the X-ray light curve at $t_{\text {jet }} \approx 6$ days, and thus derive a jet opening angle of $\theta_{\text {jet }} \approx 4^{\circ}$, yielding a beaming-corrected kinetic energy and radiated $\gamma$-ray energy of $E_{\mathrm{K}} \approx 4 \times 10^{50} \mathrm{erg}$ and $E_{\gamma} \approx 1.3 \times 10^{51} \mathrm{erg}\left(1-10^{4} \mathrm{keV}\right.$, rest frame), respectively. Consistency arguments connecting the forward shocks and reverse shocks suggest a deceleration time of $t_{\mathrm{dec}} \approx 460 \mathrm{~s} \approx T_{90}$, a Lorentz factor of $\Gamma\left(t_{\mathrm{dec}}\right) \approx 330$, and a reverse-shock-to-forward-shock fractional magnetic energy density ratio of $R_{\mathrm{B}} \equiv \epsilon_{\mathrm{B}, \mathrm{RS}} / \epsilon_{\mathrm{B}, \mathrm{FS}} \approx 8$. Our study highlights the power of rapid-response radio observations in the study of the properties and dynamics of $\gamma$-ray burst ejecta.
\end{abstract}

Key words: gamma-ray burst: general - gamma-ray burst: individual (GRB 160509A)

Supporting material: machine-readable table

\section{INTRODUCTION}

Long duration $\gamma$-ray bursts (GRBs) are produced during the catastrophic collapse of massive stars (MacFadyen \& Woosley 1999), their immense luminosity likely powered by relativistic outflows launched from a compact central engine (Piran 2005). However, the nature of the central engine launching the outflow and the mechanism producing the collimated, relativistic jet remain two urgent open questions, with models ranging from jets dominated by baryons or by Poynting flux, and those with nascent black holes or magnetars providing the central engine (see Kumar \& Zhang 2015, for a review).

A direct means of probing the outflow and thus the nature of the central engine is via the study of synchrotron radiation from the reverse shock (RS), expected when the ejecta first begin to interact with the surrounding medium (Meszaros \& Rees 1993; Sari \& Piran 1999). Consistency arguments between the synchrotron spectrum of the forward shock (FS) and the RS at the time the RS has just crossed the ejecta (the deceleration

\footnotetext{
${ }^{13}$ Einstein Fellow.
}

time, $t_{\mathrm{dec}}$ ) allow a measurement of the ejecta Lorentz factor and the ejecta magnetization, i.e., the ratio of the fractional magnetic field energy density of the RS-shocked ejecta to that of the FS-shocked circumburst medium.

Theoretically predicted to produce optical flashes on $\sim$ hour timescales, RSs were expected to be easily observable with the rapid X-ray localization enabled by Swift. However, this signature has only been seen in a few cases in the Swift era, despite optical follow-up observations as early as a few minutes after $\gamma$-ray triggers (see Japelj et al. 2014, for a review). The dearth of bright optical flashes suggests RS emission may instead be easier to observe at longer wavelengths (Mundell et al. 2007; Laskar et al. 2013; Kopac et al. 2015). We have therefore initiated a program at the Karl G. Jansky Very Large Array (VLA) for radio RS studies, and here present the detection of an RS in the Fermi GRB 160509A. Combining our radio observations with X-ray data from Swift and groundbased optical/near-infrared (NIR) observations, we perform detailed modeling of the afterglow in a robust statistical framework to derive the properties of the relativistic ejecta. Following on GRB 130427A (Laskar et al. 2013; Perley et al. 2014), this is the second GRB where multi-frequency 
radio observations enable detailed characterization of the RS emission. All magnitudes are in the AB system (Oke \& Gunn 1983), times are relative to the Large Area Telescope (LAT) trigger time, and uncertainties are reported at $68 \%(1 \sigma)$, unless otherwise noted.

\section{GRB PROPERTIES AND OBSERVATIONS}

\subsection{High-energy: Fermi}

GRB 160509A was discovered by the Fermi LAT (Atwood et al. 2009) on 2016 May 09 at 08:59:04.36 UTC (Longo et al. 2016). The burst also triggered the Fermi Gamma-ray Burst Monitor (GBM; Roberts et al. 2016). The burst duration in the $50-300 \mathrm{keV}$ GBM band is $T_{90}=369.7 \pm 0.8 \mathrm{~s}$ with a $10 \mathrm{keV}-1 \mathrm{MeV}$ fluence of $(1.790 \pm 0.002) \times 10^{-4} \mathrm{erg} \mathrm{cm}^{-2}$.

\subsection{X-Ray: Swift}

The Swift X-ray Telescope (XRT; Burrows et al. 2005) began tiled observations of the LAT error circle $2 \mathrm{hr}$ after the GRB. A fading X-ray transient was discovered at $\mathrm{R}$. A. $=20^{\mathrm{h}} 47^{\mathrm{m}} 00^{\mathrm{s}} .72$, decl. $=+76^{\mathrm{d}} 06^{\prime} 28$ ! $^{\prime \prime}$ (J2000), with an uncertainty radius of 1."5 (90\% containment; Evans 2016; Kennea 2016; Kennea et al. 2016). ${ }^{14}$ The count rate light curve exhibits a break at $\approx 4 \times 10^{4} \mathrm{~s}$. We checked for spectral evolution across the break, by extracting XRT PC-mode spectra using the on-line tool on the Swift website (Evans et al. $2007,2009)^{15}$ in the intervals $7.3 \times 10^{3} \mathrm{~s}$ to $3.7 \times 10^{4} \mathrm{~s}$ (spectrum 1) and $4.3 \times 10^{4} \mathrm{~s}$ to $1.3 \times 10^{6} \mathrm{~s}$ (spectrum 2$)$. We employ HEASOFT (v6.18) and the corresponding calibration files to fit the spectra, assuming a photoelectrically absorbed power-law model with the Galactic neutral hydrogen absorption column fixed at $N_{\mathrm{H}, \mathrm{Gal}}=2.12 \times 10^{21} \mathrm{~cm}^{-2}$ (Willingale et al. 2013), and tying the value of the intrinsic absorption in the host galaxy, $N_{\mathrm{H} \text {,int }}$, to be the same between the two spectra since we do not expect any evolution in the intrinsic absorption with time. We find only marginal evidence for spectral evolution, with $\Gamma=2.01 \pm 0.05$ in the first spectrum and $\Gamma=2.12 \pm 0.05$ in the second. Fixing the two epochs to have the same spectral index, we obtain $\Gamma_{\mathrm{X}}=$ $2.07 \pm 0.04$ and an intrinsic absorption column, $N_{\mathrm{H} \text {,int }}=$ $(1.52 \pm 0.13) \times 10^{22} \mathrm{~cm}^{-2}$. We use this value of $\Gamma_{\mathrm{X}}$ (corresponding to a spectral index ${ }^{16}$ of $\beta_{X}=-1.07 \pm$ $0.04)$ and an associated counts-to-flux ratio of $6.5 \times$ $10^{-11} \mathrm{erg} \mathrm{cm}^{-2} \mathrm{~s}^{-1} \mathrm{ct}^{-1}$ to convert the count-rate to flux density, $f_{\nu}$ at $1 \mathrm{keV}$.

\subsection{Optical/NIR}

Ground-based observations at Gemini-North beginning at $5.75 \mathrm{hr}$ uncovered a faint source $\left(r^{\prime}=23.52 \pm 0.15 \mathrm{mag}\right.$, $\left.z^{\prime}=21.35 \pm 0.30 \mathrm{mag}\right)$ consistent with the XRT position (Levan et al. 2016). Subsequent observations by the Discovery Channel Telescope $(\mathrm{DCT}) \approx 1.03$ days after the LAT trigger showed the source had faded since the Gemini observations, confirming it as the afterglow (Cenko et al. 2016). The red color in the Gemini observations, $r^{\prime}-z^{\prime} \approx 2.1$ mag indicated a high redshift or a significant amount of dust extinction within the host galaxy.

\footnotetext{
14 http://www.swift.ac.uk/xrt_positions/00020607/

15 http://www.swift.ac.uk/xrt_spectra/00020607/

${ }^{16}$ We use the convention $f_{\nu} \propto t^{\alpha} \nu^{\beta}$.
}

Table 1

GRB 160509A: Log of VLA Observations

\begin{tabular}{lcc}
\hline \hline $\begin{array}{l}\Delta t \\
\text { (days) }\end{array}$ & $\begin{array}{c}\text { Frequency } \\
(\mathrm{GHz})\end{array}$ & $\begin{array}{c}\text { Flux Density } \\
(\mu \mathrm{Jy})\end{array}$ \\
\hline 0.351 & 8.5 & $43.8 \pm 29.1$ \\
0.351 & 11.0 & $50.6 \pm 27.4$ \\
0.363 & 5.0 & $78.2 \pm 23.9$ \\
0.363 & 7.4 & $90.8 \pm 18.6$ \\
\hline
\end{tabular}

(This table is available in its entirety in machine-readable form.)

Gemini-North $J$ - and $K$-band imaging at $\approx 1.2$ days revealed an NIR counterpart with $J \sim 16.6 \mathrm{mag}$ and $K \sim 19.7 \mathrm{mag}$ (Vega magnitudes; Tanvir et al. 2016). ${ }^{17}$ Spectroscopic observations with Gemini-North at $\approx 1.2$ days yielded a single emission line identified as [O II] $3727 \AA$ at $z=1.17$, other identifications being ruled out by the absence of other lines in the spectrum (Tanvir et al. 2016). At this redshift, the inferred isotropic equivalent $\gamma$-ray energy in the $1-10^{4} \mathrm{keV}$ rest-frame energy band is $E_{\gamma \text {,iso }}=(5.76 \pm 0.05) \times 10^{53} \mathrm{erg}$.

We observed GRB 160509A using Keck-I/LRIS (Oke et al. 1995 ) beginning at $\approx 28.2$ days in $g$ - and $R$-band with integration times of $972 \mathrm{~s}$ and $900 \mathrm{~s}$, respectively. We calibrated the data using a custom LRIS pipeline, and performed photometry using Starfinder (Diolaiti et al. 2000) relative to SDSS stars in the field, obtaining $g^{\prime}=25.39 \pm$ $0.12 \mathrm{mag}$ and $r^{\prime}=24.18 \pm 0.35 \mathrm{mag}$ at 28.19 days.

\subsection{Radio}

We observed the afterglow with the VLA starting at 0.36 days. We tracked the flux density of the afterglow over multiple epochs spanning $1.2-33.5 \mathrm{GHz}$, using 3C48, 3C286, and $3 \mathrm{C} 147$ as flux and bandpass calibrators, and J2005+7752 as the gain calibrator. We carried out data reduction using the Common Astronomy Software Applications (CASA), and list the results of our VLA monitoring campaign in Table 1.

\section{MULTI-WAVELENGTH MODELING}

\subsection{Basic Considerations}

We interpret the observed behavior of the afterglow from radio to X-rays in the framework of the standard synchrotron model, described by three break frequencies (the self-absorption frequency, $\nu_{\mathrm{a}}$, the characteristic synchrotron frequency, $\nu_{\mathrm{m}}$, and the cooling frequency, $\nu_{\mathrm{c}}$ ) and an overall flux normalization, allowing for two possibilities for the density profile of the circumburst medium: the interstellar medium (ISM) profile ( $\rho=$ const; Sari et al. 1998) and the wind profile $\left(\rho \propto r^{-2}\right.$; Chevalier \& Li 2000).

\subsubsection{X-Rays-Location of $\nu_{\mathrm{c}}$ and a Jet Break}

We fit the Swift XRT light curve as a power-law with two temporal breaks. The first break occurs at $t_{\mathrm{b}, 1}=0.37 \pm$ 0.14 days when the decline rate steepens from $\alpha_{\mathrm{X}, 1}=$ $-0.51 \pm 0.12$ to $\alpha_{\mathrm{X}, 2}=-1.27 \pm 0.11 \quad\left(\Delta \alpha_{12}=-0.76 \pm\right.$ $0.17)$. This steepening does not have a simple explanation in the standard synchrotron model (for instance, the passage of $\nu_{\mathrm{c}}$ results in a steepening of the light curve by only $\Delta \alpha=-0.25$ ).

\footnotetext{
17 In the absence of reported uncertainties, we assume an uncertainty of $0.3 \mathrm{mag}$, corresponding to a $3 \sigma$ detection.
} 
It is possible that the X-ray data before $t_{\mathrm{b}, 1}$ are part of a plateau phase, which is commonly observed among GRB X-ray afterglows (Nousek et al. 2006), and we therefore do not consider the X-ray observations before $\approx 0.35$ days in the remainder of our analysis.

At $t_{\mathrm{b}, 2}=5.4 \pm 2.3$ days, the light curve steepens again to $\alpha_{\mathrm{X}, 3}=-2.2 \pm 0.3\left(\Delta \alpha_{23}=-0.9 \pm 0.3\right)$, suggestive of a jet break. Since $\nu_{\mathrm{m}} \propto t^{-1.5}$ is expected to be below the $\mathrm{X}$-ray band at this time and the post-break decay rate at $\nu>\nu_{\mathrm{m}}$ is $t^{-p}$, we determine the energy index of non-thermal electrons, $p \approx 2.2$ (Sari et al. 1999). For this value of $p$, we expect a spectral slope of $\beta_{\mathrm{X}} \approx-1.1$ or $\beta_{\mathrm{X}} \approx-0.6$ for $\nu_{\mathrm{c}}<\nu_{\mathrm{X}}$ and $\nu_{\mathrm{c}}>\nu_{\mathrm{X}}$, respectively. The measured $\mathrm{X}$-ray spectral index of $\beta_{\mathrm{X}}=-1.07 \pm 0.04$ requires the former, whereupon we expect $\alpha_{\mathrm{X}}=(2-3 p) / 4 \approx-1.2$. This is consistent with the measured value of $\alpha_{\mathrm{X}, 2}=-1.27 \pm 0.11$. Thus, we conclude that the X-ray light curve and spectrum are both consistent with $p \approx 2.2$ and $\nu_{\mathrm{c}}<\nu_{\mathrm{X}}$. We note that in this regime the $\mathrm{X}$-ray light curve does not distinguish between the ISM and wind models.

\subsubsection{Optical/NIR - Extinction and Host Flux}

At the time of the Gemini $z^{\prime}$ - and $r^{\prime}$-band observations (0.24 days), the $\mathrm{X}$-ray to $z^{\prime}$-band spectral index is flat, $\beta_{\mathrm{ox}}=-0.11 \pm 0.06$, while the $z^{\prime}-r^{\prime}$ spectral index is extremely steep, $\beta_{\mathrm{zr}}=-5.4 \pm 1.1$. Given the moderate redshift of the burst, the only explanation for these observations is a large amount of extinction along the sight-line through the GRB host galaxy, suppressing the optical flux. On the other hand, the spectral index between the DCT $r^{\prime}$ - and $g$-band observations at $\approx 1$ day is $\beta_{\mathrm{gr}}=-1.9 \pm 0.6$, significantly shallower than $\beta_{\mathrm{zr}}$, while the $r^{\prime}$-band light curve before $\approx 1$ day declines as $\alpha_{\mathrm{r}}=-0.33 \pm 0.02$, shallower than expected in the standard afterglow model. Together, these observations indicate a significant contribution to the afterglow photometry from the host galaxy. This is confirmed by our Keck $g$ - and $R$-band observations at $\approx 28$ days, which yield flux densities similar to the DCT observations at $\approx 1$ days. We find that modeling the $r^{\prime}$ band light curve as a sum of a power-law and a constant yields $\alpha_{\mathrm{r}}=-1.09 \pm 0.45$, with the additive constant $f_{\nu, \mathrm{r}}=0.75 \pm 0.10 \mu \mathrm{Jy}$. We note that whereas the light curve decay rate at $\nu_{\mathrm{m}}<\nu<\nu_{\mathrm{c}}$ is expected to provide diagnostic power for the circumburst density profile, the paucity of optical data and the large uncertainty in the optical decay rate for this event preclude such a discrimination. In the detailed modeling (Section 3.3) we fit for the host galaxy flux density in all optical/NIR filters, together with the optical extinction along the line of sight through the host.

\subsubsection{Radio-Multiple Components}

The radio spectral energy distribution (SED) at 4.06 days exhibits a clear peak at $\approx 8.4 \mathrm{GHz}$ with a flux density of $\approx 1.2$ $\mathrm{mJy}$. At this time, the measured X-ray flux density is $f_{\nu, \mathrm{X}}=(6.3 \pm 1.9) \times 10^{-4} \mathrm{mJy}$. Fitting the radio data with a broken power-law and extrapolating to the X-rays, we find that the expected X-ray flux density is at least two orders of magnitude lower than observed (Figure 1). This suggests that the radio and X-ray emissions at 4.06 days arise from separate processes. Further, we note that the radio spectral index above $10 \mathrm{GHz}$ at 10 days is $\beta_{\text {radio }}(10$ days $)=0.1 \pm 0.2$, in contrast to the spectral index above the peak at 4.06 days, $\beta_{\text {radio }}$ (4.06 days) $=-0.79 \pm 0.02$. Since such a hardening of the spectral index is not expected in the standard synchrotron model, we propose that the radio peak at 4.06 days has faded to reveal a fainter underlying component at 10 days. We show this underlying emission to be consistent with the FS in Section 3.3.

To summarize, the X-ray spectral index and light curve are consistent with an FS origin for the X-ray emission with $p \approx 2.2$ and $\nu_{\mathrm{c}}<\nu_{\mathrm{X}}$. The radio spectrum at 4.06 days cannot be extrapolated to match the observed X-ray flux at this time, suggesting that the radio and X-ray emission arise from separate processes. The radio peak at 4.06 days fades to reveal an underlying power-law continuum, which we ascribe to the FS. Finally, there is insufficient information in the afterglow observations to constrain the circumburst density profile.

\subsection{The Reverse Shock}

We construct a model SED for the radio to X-ray emission at 1.13 days comprising two emission components: (1) an FS (Section 3.3), which peaks between the radio and optical bands, fits the NIR to X-ray SED, and provides negligible contribution in the radio band, and (2) an RS (this section), which fits the radio SED and provides negligible contribution at higher frequencies. The synchrotron parameters of the RS are listed in Table 2. We find that this combined RS plus FS model completely describes the observed SED at 1.13 days (Figure 1).

We evolve both emission components to the epochs of our radio observations. The evolution of the RS spectrum depends on whether the shock is Newtonian or relativistic in the frame of the unshocked ejecta, and is determined by the evolution of the ejecta Lorentz factor with radius quantified by the parameter $g: \Gamma \propto R^{-g} \propto t^{-g /(1+2 g)}$. This was first measured observationally for GRB 130427A, where a value of $g \approx 5$ was inferred for a Newtonian RS (Laskar et al. 2013). We find that evolving the RS SED for GRB 160509A with $g \approx 2$ matches the observed radio spectrum well from 0.36 to 10 days. This value of $g$ closely matches the predicted value of $g \approx 2.2$ from numerical calculations of the RS evolution for a Newtonian RS (Kobayashi \& Sari 2000). A value of $g \approx 3$ expected for a relativistic RS is ruled out by the observed evolution of the radio SED, providing the second direct measurement of $g$, and the first observational confirmation of the numerical theory.

The radio peak ascribed to the $\mathrm{RS}$ emission fades faster than expected from the $\mathrm{RS}$ model after $\approx 5$ days. We note that this coincides with the time of the jet break in the X-ray light curve (Section 3.1). The standard FS jet break is a combination of geometrical effects that take place when the FS Lorentz factor, $\Gamma \approx \theta_{\text {jet }}^{-1}$ : the observer sees the edge of the jet and the swept-up material begins to expand sideways (Rhoads 1999; De Colle et al. 2012; Granot \& Piran 2012). In the case of the RS, the ejecta internal energy drops rapidly after the RS crossing and the local sound velocity in the ejecta is expected to be subrelativistic. Thus, we expect the lateral expansion to be fairly slow, resulting in no change in the dynamics or the scaling of the RS break frequencies across the jet break. The geometric effect is expected to dominant, resulting in a change in the RS peak flux scaling by $\Gamma_{\mathrm{RS}}^{2}$ at $t_{\mathrm{jet}}$. Setting the RS jet break time to 5.2 days as derived from a preliminary fit to the FS (Section 3.3), we find that the resultant evolution of the RS SED fits all subsequent radio observations well (Figure 2).

Finally, we note that $\nu_{\mathrm{c}, \mathrm{RS}}$ passes through the NIR at $\approx 3 \times 10^{-2}$ days in this model. After this time, we do not 

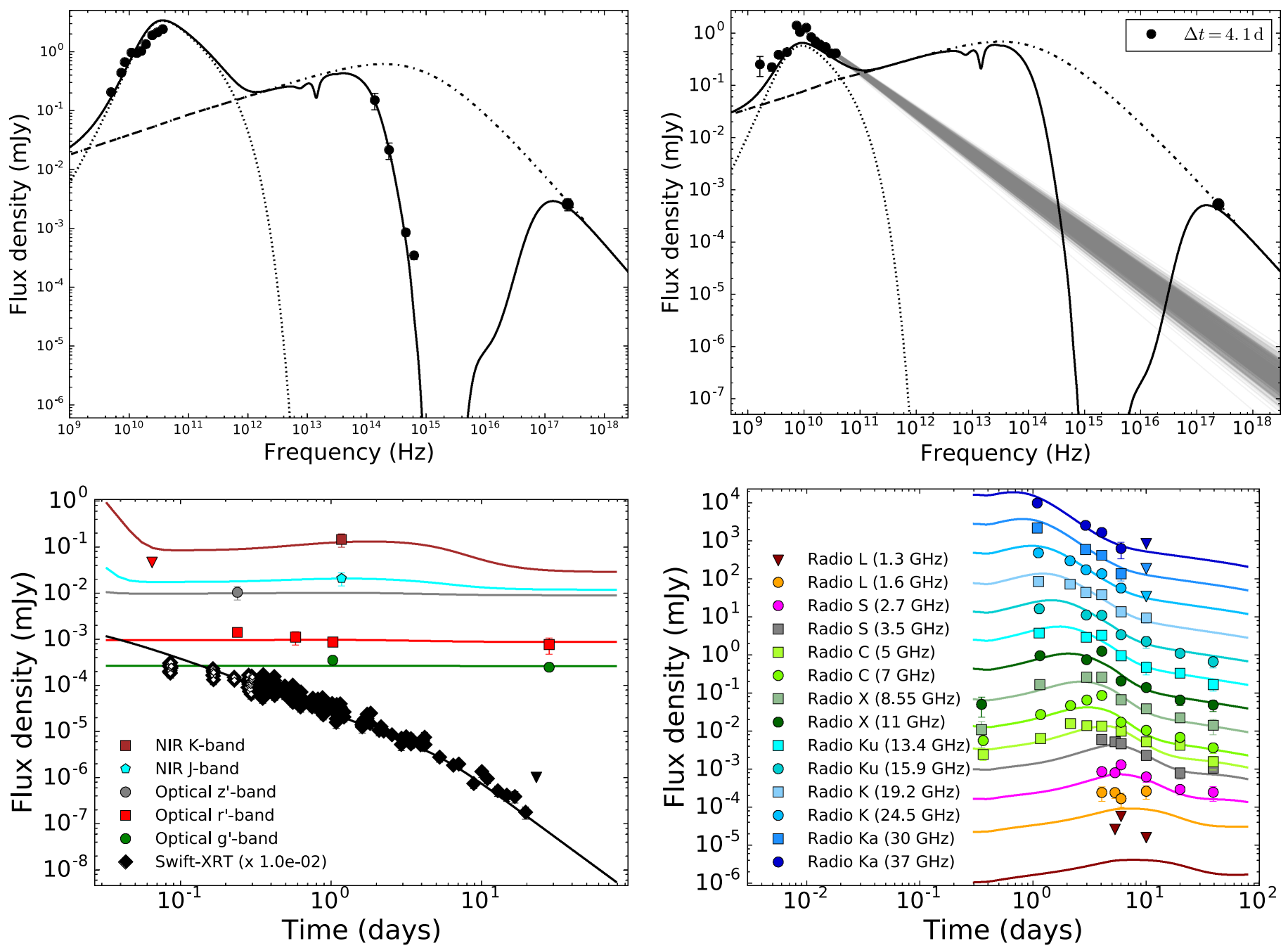

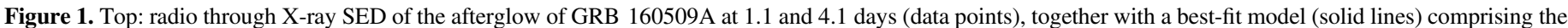

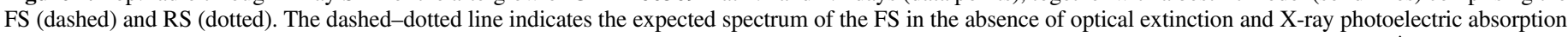

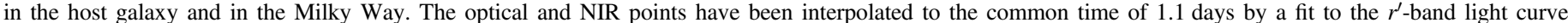

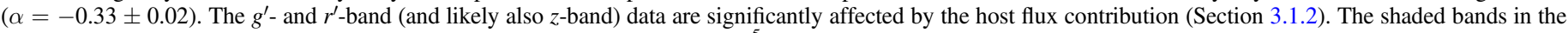

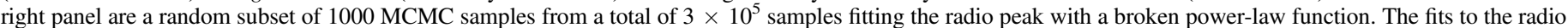

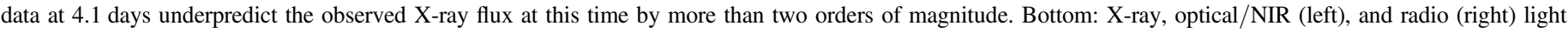

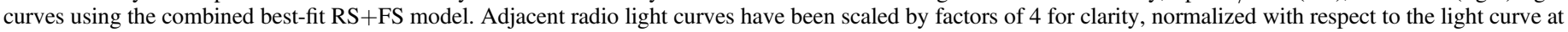
$11 \mathrm{GHz}$.

expect observable RS emission in the optical/NIR. This is consistent with the earliest available $R$-band observation $\left(R<19.5 \mathrm{mag}\right.$ at $6.5 \times 10^{-2}$ days; Izzo et al. 2016), and with all subsequent optical/NIR data.

\subsection{The Forward Shock}

To model the FS emission we employ the framework of synchrotron radiation from relativistic shocks, including the effects of inverse Compton cooling (Sari \& Esin 2001; Granot $\&$ Sari 2002). The parameters of the fit are the kinetic energy $\left(E_{\mathrm{K} \text {,iso }}\right)$, the density $\left(n_{0}\right)$, the electron energy index $(p)$, and the fraction of the shock energy given to electrons $\left(\epsilon_{\mathrm{e}}\right)$ and magnetic fields $\left(\epsilon_{\mathrm{B}}\right)$. We use the Small Magellanic Cloud (SMC) extinction curve to model the extinction $\left(A_{\mathrm{V}}\right)$ in the GRB host galaxy (Pei 1992), and include the flux density of the host in the $g r z J K$ bands $\left(f_{\nu \text {,host }}\right)$, together with the jet break time $\left(t_{\text {jet }}\right)$, as additional free parameters.
The afterglow observations in this case do not allow us to directly determine the circumburst density profile, and both ISM and wind-like environments have been inferred for GRBs in the past (e.g., Panaitescu \& Kumar 2002; Yost et al. 2003; Cenko et al. 2010, 2011; Schulze et al. 2011). However, we find that consistency arguments between the FS and RS SEDs at the deceleration time provide meaningful results in the ISM case, but not in the wind case. We therefore focus on the ISM model in the remainder of the article, and discuss the wind model briefly in Section 4.3.

We fit all available photometry with a combination of the RS and FS contributions. A least-squares analysis provides the starting point, using which we find an FS jet break time of $t_{\text {jet }} \approx 5.2$ days. We fix the RS jet break time to this value. To efficiently sample parameter space and to uncover correlations between the parameters, we then carry out a Markov chain Monte Carlo (MCMC) analysis using EMCEE (ForemanMackey et al. 2013). Our analysis methods are described in detail by Laskar et al. (2014). The resultant marginalized 
Table 2

Model Parameters

\begin{tabular}{lc}
\hline \hline Parameter & Value \\
\hline \multicolumn{3}{c}{ Reverse Shock } \\
\hline$\nu_{\mathrm{a}, \mathrm{RS}}$ & \\
$\nu_{\mathrm{m}, \mathrm{RS}}$ & $2.5 \times 10^{10} \mathrm{~Hz}$ \\
$\nu_{\mathrm{c}, \mathrm{RS}}$ & $1.5 \times 10^{10} \mathrm{~Hz}$ \\
$f_{\nu, \mathrm{m}, \mathrm{RS}}$ & $4 \times 10^{11} \mathrm{~Hz}$ \\
\hline
\end{tabular}

\begin{tabular}{|c|c|}
\hline \multicolumn{2}{|c|}{ Forward Shock (ISM) } \\
\hline$p$ & $2.39 \pm 0.03$ \\
\hline$\epsilon_{\mathrm{e}}$ & $0.84_{-0.08}^{+0.06}$ \\
\hline$\epsilon_{\mathrm{B}}$ & $0.11_{-0.05}^{+0.07}$ \\
\hline$n_{0}$ & $(8.6 \pm 2.2) \times 10^{-4} \mathrm{~cm}^{-3}$ \\
\hline$E_{\mathrm{K}, \text { iso }}$ & $\left(18.7_{-2.6}^{+5.4}\right) \times 10^{52} \mathrm{erg}$ \\
\hline$A_{\mathrm{V}}$ & $3.35_{-0.07}^{+0.08} \mathrm{mag}$ \\
\hline$t_{\text {jet }}$ & $5.7_{-0.5}^{+0.6}$ days \\
\hline$f_{\nu, \text { host }, \mathrm{g}}$ & $0.29 \mu \mathrm{Jy}$ \\
\hline$f_{\nu, \text { host } \mathrm{r}}$ & $0.88 \mu \mathrm{Jy}$ \\
\hline$f_{\nu, \text { host }, \mathrm{z}}$ & $9.0 \mu \mathrm{Jy}$ \\
\hline$f_{\nu, \text { host }, \mathrm{J}}$ & $11.9 \mu \mathrm{Jy}$ \\
\hline$f_{\nu, \text { host, } \mathrm{K}}$ & $28.8 \mu \mathrm{Jy}$ \\
\hline$\theta_{\text {jet }}$ & $3.89_{-0.16}^{+0.14 \circ}$ \\
\hline$E_{\mathrm{K}}^{\mathrm{a}}$ & $\left(4.4_{-0.7}^{+1.1}\right) \times 10^{50} \mathrm{erg}$ \\
\hline$E_{\gamma}^{\mathrm{a}, \mathrm{b}}$ & $(1.3 \pm 0.1) \times 10^{51} \mathrm{erg}$ \\
\hline$\nu_{\mathrm{a}, \mathrm{FS}}$ & $1.2 \times 10^{7} \mathrm{~Hz}$ \\
\hline$\nu_{\mathrm{m}, \mathrm{FS}}$ & $8.7 \times 10^{14} \mathrm{~Hz}$ \\
\hline$\nu_{\mathrm{c}, \mathrm{FS}}$ & $3.2 \times 10^{15} \mathrm{~Hz}$ \\
\hline$f_{\nu, \mathrm{m}, \mathrm{FS}}$ & $1.6 \mathrm{mJy}$ \\
\hline
\end{tabular}

\begin{tabular}{lc}
\hline \multicolumn{3}{c}{ Forward Shock (wind) } \\
\hline$p$ & 2.11 \\
$\epsilon_{\mathrm{e}}$ & 0.60 \\
$\epsilon_{\mathrm{B}}$ & 0.40 \\
$A_{*}$ & $5.3 \times 10^{-3} \mathrm{~cm}^{-3}$ \\
$E_{\mathrm{K}, \text { iso }}$ & $3.0 \times 10^{53} \mathrm{erg}$ \\
$A_{\mathrm{V}}$ & $4.1 \mathrm{mag}$ \\
$t_{\text {jet }}$ & $5.5 \mathrm{days}$ \\
$f_{\nu, \text { host }, g}$ & $0.26 \mu \mathrm{Jy}$ \\
$f_{\nu, \text { host }, r}$ & $0.86 \mu \mathrm{Jy}$ \\
$f_{\nu, \text { host }, z}$ & $7.2 \mu \mathrm{Jy}$ \\
$f_{\nu, \text { host }, J}$ & $15.7 \mu \mathrm{Jy}$ \\
$f_{\nu, \text { host }, K}$ & $66.4 \mu \mathrm{Jy}$ \\
$\theta_{\text {jet }}$ & $1.6^{\circ}$ \\
$E_{\mathrm{K}}{ }^{\mathrm{a}}$ & $1.3 \times 10^{50} \mathrm{erg}$ \\
$E_{\gamma}{ }^{\mathrm{a}, \mathrm{b}}$ & $(2.2 \pm 0.2) \times 10^{50} \mathrm{erg}$ \\
$\nu_{\mathrm{a}, \mathrm{FS}}$ & $1.2 \times 10^{7} \mathrm{~Hz}$ \\
$\nu_{\mathrm{m}, \mathrm{FS}}$ & $1.2 \times 10^{14} \mathrm{~Hz}$ \\
$\nu_{\mathrm{c}, \mathrm{FS}}$ & $1.1 \times 10^{16} \mathrm{~Hz}$ \\
$f_{\nu, \mathrm{m}, \mathrm{FS}}$ & 1.6 \\
\hline & \\
&
\end{tabular}

Notes. All frequencies and flux densities in this table are calculated at 1 day. The host flux density measurements are corrected for Milky Way extinction and are presented for a representative model.

${ }^{\text {a }}$ Corrected for beaming.

b $1-10^{4} \mathrm{keV}$, rest frame.

posterior density functions are summarized in Table 2 and Figure 3. Correlation functions between the four physical parameters are plotted in Figure 4. In our best-fit model
( $\chi^{2}=16.4$ for 12 degrees of freedom), the FS transitions from fast cooling to slow cooling at $\approx 0.3$ days, while the Compton $Y$-parameter is $\approx 2.4$, indicating that inverse-Compton cooling is moderately significant.

\section{DISCUSSION}

\subsection{Self-consistency of RS and FS models}

In the standard synchrotron model, the break frequencies of the $\mathrm{RS}$ and FS spectra are expected to be related at $t_{\mathrm{dec}}: \nu_{\mathrm{c}, \mathrm{RS}} /$ $\nu_{\mathrm{c}, \mathrm{FS}} \sim R_{\mathrm{B}}^{-3 / 2}, \quad \nu_{\mathrm{m}, \mathrm{RS}} / \nu_{\mathrm{m}, \mathrm{FS}} \sim R_{\mathrm{B}}^{1 / 2} \Gamma_{0}^{-2}$, and $f_{\nu, \mathrm{m}, \mathrm{RS}} / f_{\nu, \mathrm{m}, \mathrm{FS}} \sim$ $\Gamma_{0} R_{\mathrm{B}}^{1 / 2}$, where $\Gamma_{0}$ is the bulk Lorentz factor at $t_{\mathrm{dec}}$, and $R_{\mathrm{B}} \equiv \epsilon_{\mathrm{B}, \mathrm{RS}} / \epsilon_{\mathrm{B}, \mathrm{FS}}$ is the ejecta magnetization parameter (Gomboc et al. 2008; Harrison \& Kobayashi 2013). The three relations above then provide three constraints that can be solved exactly for $t_{\mathrm{dec}}, \Gamma_{0}$, and $R_{\mathrm{B}}$. For our best-fit FS $+\mathrm{RS}$ model, we find $t_{\mathrm{dec}} \approx$ $460 \mathrm{~s} \approx T_{90}, \quad \Gamma_{0} \approx 330$, and $R_{\mathrm{B}} \approx 8$. We note that the derived values of $E_{\mathrm{K} \text {,iso }}, n_{0}, \theta_{\text {jet }}$, and $\Gamma_{0}$ can be used to derive a jet break time for the RS using the relation, $t_{\text {jet }}=$ $110(1+z)\left(E_{\mathrm{K}, \text { iso }, 52} / n_{0}\right)^{1 / 3} \theta_{\text {jet }}^{5 / 2} \Gamma_{0}^{-1 / 6}$ days (Gao et al. 2013). Using the best-fit FS model, we find $t_{\text {jet,RS }} \approx 3.4$ days, which is slightly earlier than the FS jet break time, as expected. The difference between this value and our assumed value of $\approx 5.2$ days in Section 3.2 only marginally affects the fit at one of the epochs (4.06 days) in Figure 2. A fully consistent solution requires bootstrapping the $\mathrm{FS}$ and $\mathrm{RS}$ parameters together, and we defer such an analysis to future work.

\subsection{Low-density Environments and the RS}

In our previous work on GRB 130427A, we suggested that a slow-cooling $\mathrm{RS}$ is more likely to produce detectable radio emission (Laskar et al. 2013). Since $\nu_{\mathrm{c}, \mathrm{RS}} / \nu_{\mathrm{c}, \mathrm{FS}} \propto n_{0}^{-4 / 3}$ at $t_{\mathrm{dec}}$, a low-density environment may be a requisite factor for observing long-lasting RS emission (Kobayashi 2000; Resmi \& Zhang 2016). We find a low circumburst density in the context of long-lasting RS emission for GRB 160509A, leading credence to this hypothesis. However, we also note that additional considerations such as high $f_{\nu, \mathrm{m}, \mathrm{RS}}$ or late deceleration times may also contribute to stronger RS signatures; therefore, the detectability of an RS remains a complex question (Kopac et al. 2015).

\subsection{Wind Model}

Since the available afterglow observations do not distinguish strongly between a wind and ISM model, we also provide the parameters for a fiducial wind model (Table 2). For this model, the spectrum transitions from fast cooling to slow cooling at 0.17 days, and the spectral break frequencies at 1 day are within a factor of $\approx 3$ of the values derived for the ISM model in Section 3.3. We note that the value of $g \approx 2$ for the RS remains plausible in the wind environment as well and, therefore, the RS parameters derived in Section 3.2 remain reasonable. Combining the RS and FS parameters for the wind model, we find $t_{\mathrm{dec}} \approx 170 \mathrm{~s}, \Gamma_{0} \approx 34$, and $R_{\mathrm{B}} \approx 0.05$. The low value of $\Gamma_{0}$, the low inferred magnetization, and finding $t_{\mathrm{dec}} \lesssim T_{90}$, together argue against the wind model (Fenimore et al. 1993; Woods \& Loeb 1995). 

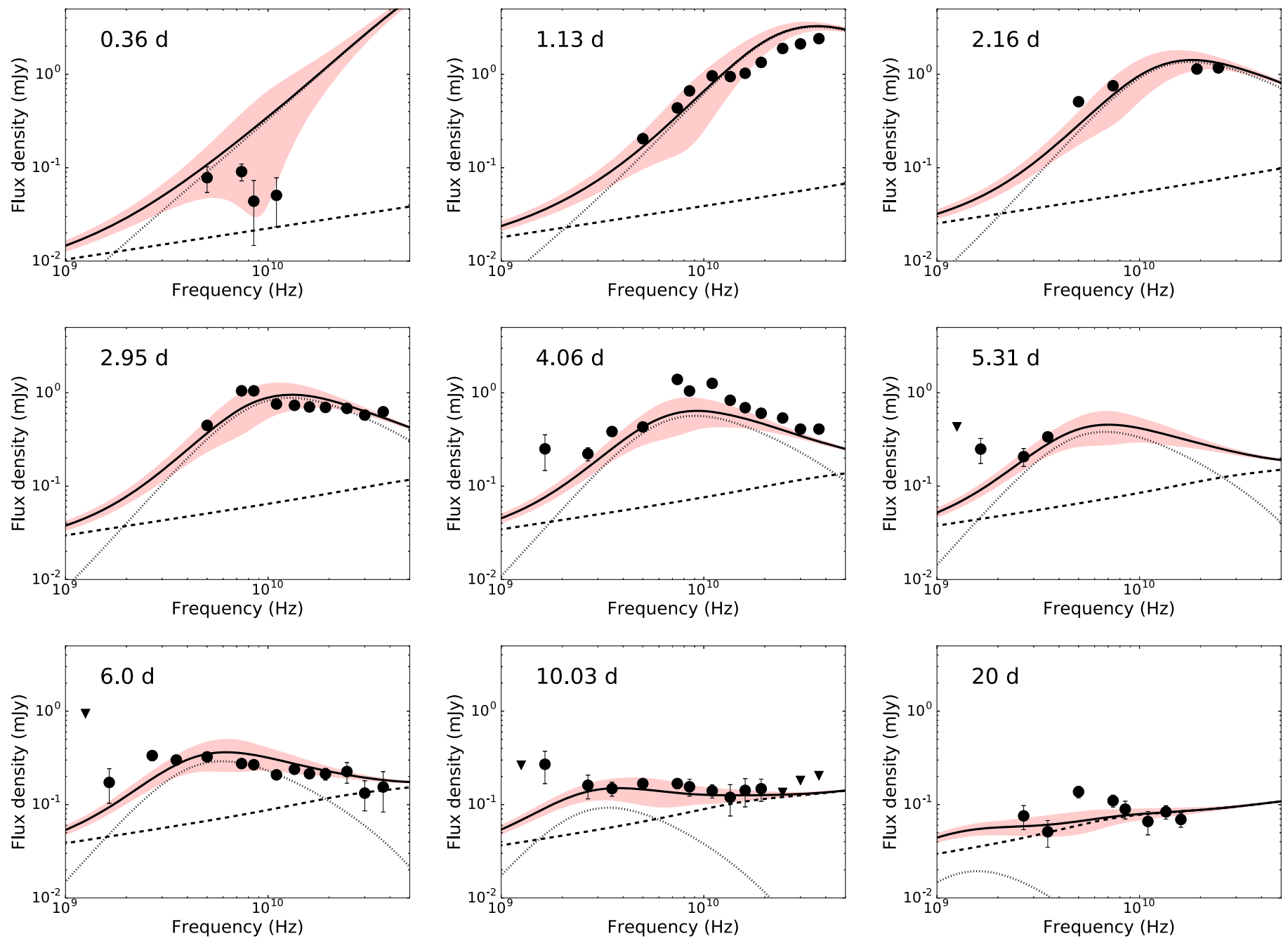

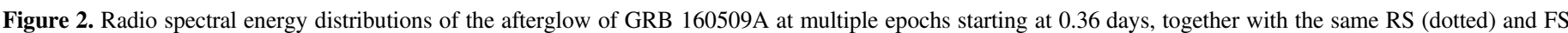

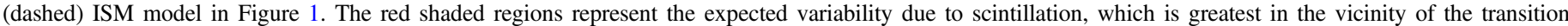
frequency along the line of sight to the $\mathrm{GRB}, \nu_{\mathrm{T}}=13.55 \mathrm{GHz}$. The radio observations up to 10.03 days are dominated by the RS.

\subsection{Neutral Hydrogen Column Density and Extinction}

A correlation between the neutral hydrogen column derived from X-ray absorption and the line-of-sight extinction, $N_{\mathrm{H}} \approx 2 \times 10^{21} \mathrm{~cm}^{-2}\left(A_{\mathrm{V}} \mathrm{mag}^{-1}\right)$, has been observed for the Milky Way (Predehl \& Schmitt 1995; Güver \& Özel 2009). However, the majority of GRB afterglows exhibit lower values of $A_{\mathrm{V}}$ than would be expected from this correlation (e.g., Galama \& Wijers 2001; Stratta et al. 2004; Zafar et al. 2010; Zauderer et al. 2013). We note that the extinction of GRB afterglows by their host galaxy is often well fit with an SMC extinction curve (as we also do here; Japelj et al. 2015). We therefore derive a corresponding correlation for the SMC using the relation between $N_{\mathrm{H}}$ and $E(B-V)$ from Welty et al. (2012) and the mean $R_{\mathrm{V}}=2.74$ for the SMC bar from Gordon et al. (2003), obtaining $\log \left(N_{\mathrm{H}} / 10^{21} \mathrm{~cm}^{-2}\right)=21.95 \pm 0.36+\log \left(A_{\mathrm{V}} \mathrm{mag}^{-1}\right)$. For $N_{\mathrm{H}} \approx 1.5 \times 10^{22} \mathrm{~cm}^{-2}$, this gives $\log \left(A_{\mathrm{V}} \mathrm{mag}^{-1}\right)=0.23 \pm$ 0.36 or $A_{\mathrm{V}}=1.7_{-1.0}^{+2.2} \mathrm{mag}$, while the MW correlation gives $A_{\mathrm{V}}=(7.6 \pm 0.7) \mathrm{mag}$. Our observed value of $A_{\mathrm{V}}=$ $3.35_{-0.07}^{+0.08} \mathrm{mag}$ is, therefore, intermediate between the values expected from the two relations.

\section{CONCLUSIONS}

We present a detailed multi-wavelength study of the FermiLAT GRB 160509A at $z=1.17$. Our VLA observations spanning 0.36-20 days after the burst clearly reveal the presence of multiple spectral components in the radio afterglow. We identify the two spectral components as arising from the FS and RS, and from a joint analysis of the two emission components, we conclude the following.

1. The RS dominates in the radio before $\approx 10$ days, and the FS dominates in the X-ray and optical/NIR.

2. The evolution of the RS spectrum requires a Lorentz factor index, $g \approx 2$, consistent with theoretical predictions for a Newtonian RS. We derive a deceleration time of $460 \mathrm{~s}$, a Lorentz factor of $\Gamma_{0} \approx 330$ at the deceleration time, and an ejecta magnetization of $R_{\mathrm{B}} \approx 8$.

3. The afterglow observations do not strongly constrain the density profile of the circumburst environment. However, the RS-FS consistency relations yield a very low Lorentz factor in the wind environment. 

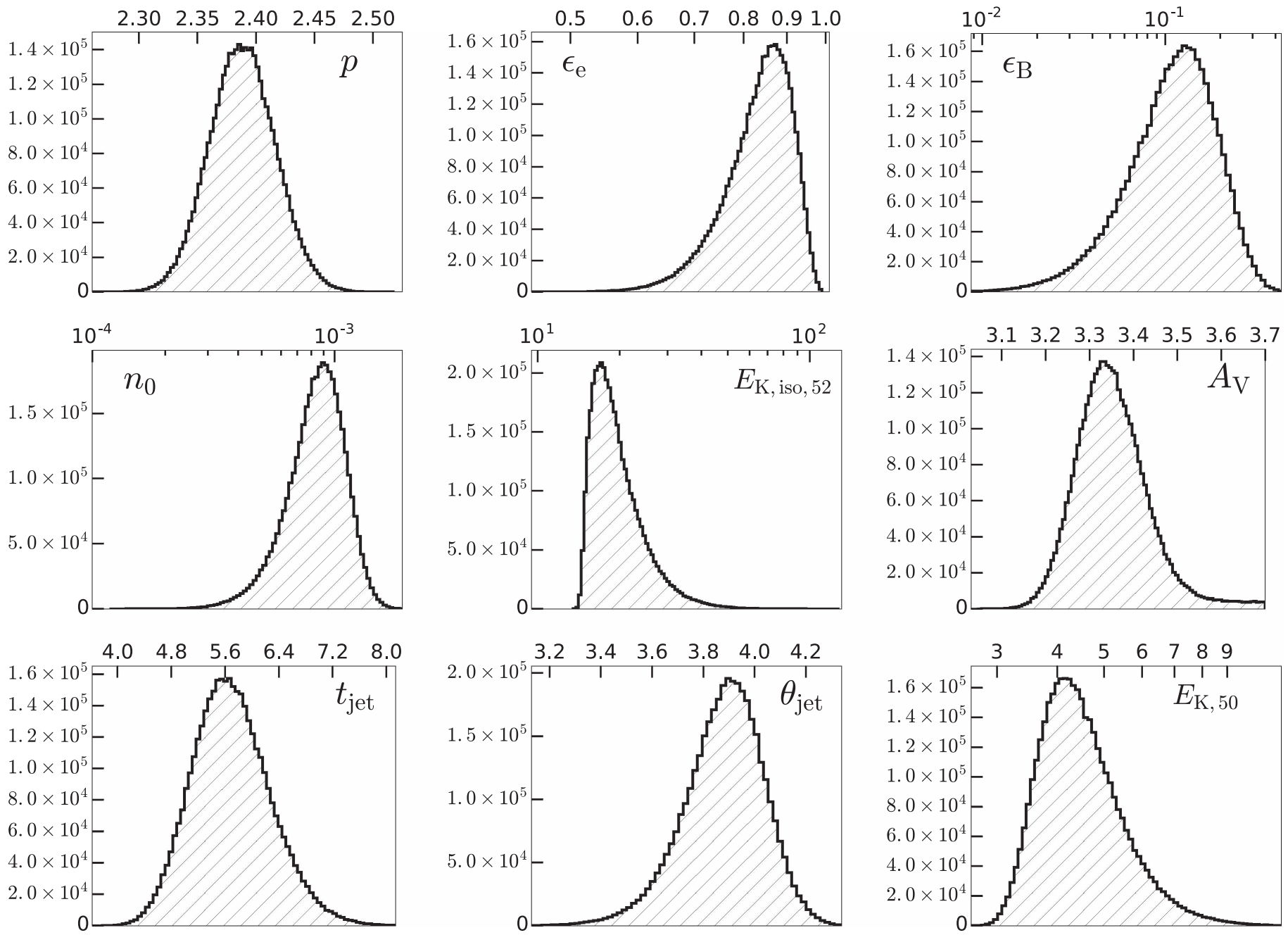

Figure 3. Marginalized posterior probability density functions of the FS parameters from MCMC simulations. We have restricted $\epsilon_{\mathrm{e}}+\epsilon_{\mathrm{B}}<1$.
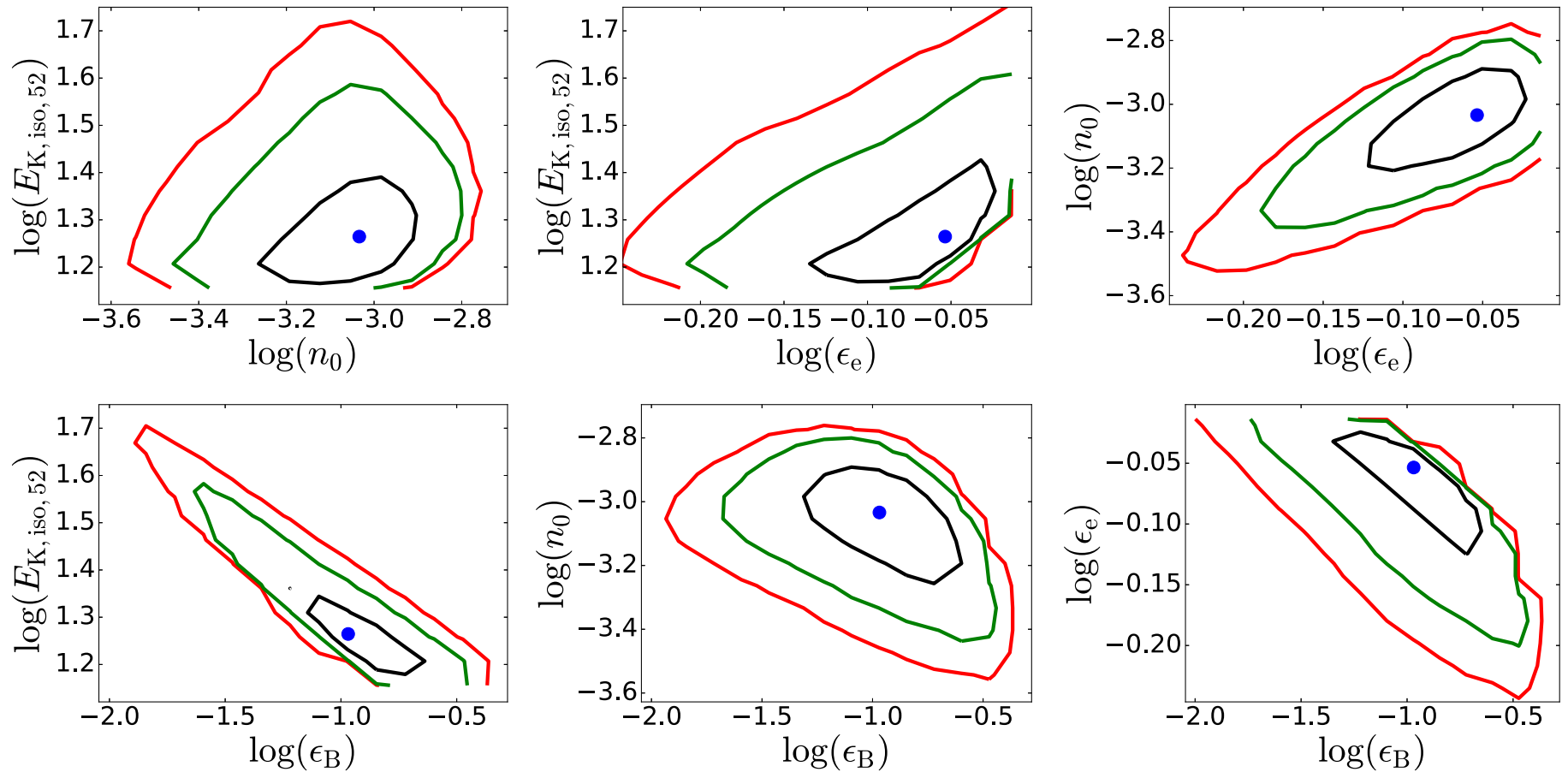

Figure 4. $1 \sigma$ (red), $2 \sigma$ (green), and $3 \sigma$ (black) contours for correlations between the physical parameters $E_{\mathrm{K}, \text { iso }}, n_{0}, \epsilon_{\mathrm{e}}$, and $\epsilon_{\mathrm{B}}$ from Monte Carlo simulations, together with the best-fit model (blue dot). We have restricted $\epsilon_{\mathrm{e}}+\epsilon_{\mathrm{B}}<1$. 
4. We derive a circumburst density of $n_{0} \approx 10^{-3} \mathrm{~cm}^{-3}$, supporting the hypothesis that a low density environment may be a requisite factor in producing a slow-cooling and long-lasting RS.

This work follows on our previous successful identification and characterization of an RS in GRB 130427A, and highlights the importance of rapid-response radio observations in the study of the properties and dynamics of GRB ejecta.

T.L. is a Jansky Fellow of the National Radio Astronomy Observatory. EB acknowledges support from NSF grant AST1411763 and NASA ADA grant NNX15AE50G. W.F. is supported by NASA through Einstein Postdoctoral Fellowship grant number PF4-150121. A.V.F.'s group at UC Berkeley has received generous financial assistance from Gary and Cynthia Bengier, the Richard and Rhoda Goldman Fund, the Christopher R. Redlich Fund, the TABASGO Foundation, NSF grant AST-1211916, and NASA/Swift grant NNX12AD73G. This work was supported in part by the NSF under grant No. PHYS-1066293; A.V.F. thanks the Aspen Center for Physics for its hospitality during the black holes workshop in 2016 June. This research has made use of data obtained through the High Energy Astrophysics Science Archive Research Center Online Service, provided by the NASA/Goddard Space Flight Center. Some of the data presented herein were obtained at the W.M. Keck Observatory, which is operated as a scientific partnership among the California Institute of Technology, the University of California, and the National Aeronautics and Space Administration, and was made possible by the generous financial support of the W.M. Keck Foundation. VLA observations were taken as part of our VLA Large Program 15A-235 (PI: E. Berger). The National Radio Astronomy Observatory is a facility of the National Science Foundation operated under cooperative agreement by Associated Universities, Inc.

\section{REFERENCES}

Atwood, W. B., Abdo, A. A., Ackermann, M., et al. 2009, ApJ, 697, 1071 Burrows, D. N., Hill, J. E., Nousek, J. A., et al. 2005, SSRv, 120, 165 Cenko, S. B., Frail, D. A., Harrison, F. A., et al. 2010, ApJ, 711, 641

Cenko, S. B., Frail, D. A., Harrison, F. A., et al. 2011, ApJ, 732, 29

Cenko, S. B., Troja, E., \& Tegler, S. 2016, GCN, 19416

Chevalier, R. A., \& Li, Z.-Y. 2000, ApJ, 536, 195

De Colle, F., Ramirez-Ruiz, E., Granot, J., \& Lopez-Camara, D. 2012, ApJ, 751,57

Diolaiti, E., Bendinelli, O., Bonaccini, D., et al. 2000, ApJ, 147, 335

Evans, P. A. 2016, GCN, 19406
Evans, P. A., Beardmore, A. P., Page, K. L., et al. 2007, A\&A, 469, 379

Evans, P. A., Beardmore, A. P., Page, K. L., et al. 2009, MNRAS, 397, 1177

Fenimore, E. E., Epstein, R. I., \& Ho, C. 1993, A\&AS, 97, 59

Foreman-Mackey, D., Hogg, D. W., Lang, D., \& Goodman, J. 2013, PASP, 125,306

Galama, T. J., \& Wijers, R. A. M. J. 2001, ApJL, 549, L209

Gao, H., Lei, W.-H., Zou, Y.-C., Wu, X.-F., \& Zhang, B. 2013, NewAR, 57,141

Gomboc, A., Kobayashi, S., Guidorzi, C., et al. 2008, ApJ, 687, 443

Gordon, K. D., Clayton, G. C., Misselt, K. A., Landolt, A. U., \& Wolff, M. J. 2003, ApJ, 594, 279

Granot, J., \& Piran, T. 2012, MNRAS, 421, 570

Granot, J., \& Sari, R. 2002, ApJ, 568, 820

Güver, T., \& Özel, F. 2009, MNRAS, 400, 2050

Harrison, R., \& Kobayashi, S. 2013, ApJ, 772, 101

Izzo, L., de Ugarte Postigo, A., \& Thoene, C. 2016, GCN, 19409

Japelj, J., Covino, S., Gomboc, A., et al. 2015, A\&A, 579, A74

Japelj, J., Kopac, D., Kobayashi, S., et al. 2014, ApJ, 785, 84

Kennea, J. A. 2016, GCN, 19407

Kennea , J. A., Roegiers, T. G. R., Osborne, J. P., \& Page, K. L. E. A. 2016, GCN, 19408

Kobayashi, S. 2000, ApJ, 545, 807

Kobayashi, S., \& Sari, R. 2000, ApJ, 542, 819

Kopac, D., Mundell, C. G., Kobayashi, S., et al. 2015, ApJ, 806, 179

Kumar, P., \& Zhang, B. 2015, PhR, 561, 1

Laskar, T., Berger, E., Tanvir, N., et al. 2014, ApJ, 781, 1

Laskar, T., Berger, E., Zauderer, B. A., et al. 2013, ApJ, 776, 119

Levan, A. J., Tanvir, N. R., Cenko, S. B., \& Perley, D. 2016, GCN, 19410

Longo, F., Bissaldi, E., Bregeon, J., et al. 2016, GCN, 19403

MacFadyen, A. I., \& Woosley, S. E. 1999, ApJ, 524, 262

Meszaros, P., \& Rees, M. J. 1993, ApJ, 405, 278

Mundell, C. G., Melandri, A., Guidorzi, C., et al. 2007, ApJ, 660, 489

Nousek, J. A., Kouveliotou, C., Grupe, D., et al. 2006, ApJ, 642, 389

Oke, J. B., \& Gunn, J. E. 1983, ApJ, 266, 713

Oke, J. B., Cohen, J. G., Carr, M., et al. 1995, PASP, 107, 375

Panaitescu, A., \& Kumar, P. 2002, ApJ, 571, 779

Pei, Y. C. 1992, ApJ, 395, 130

Perley, D. A., Cenko, S. B., Corsi, A., et al. 2014, ApJ, 781, 37

Piran, T. 2005, Rev. Mod. Phys., 76, 1143

Predehl, P., \& Schmitt, J. H. M. M. 1995, A\&A, 293, 889

Resmi, L., \& Zhang, B. 2016, ApJ, 825, 48

Rhoads, J. E. 1999, ApJ, 525, 737

Roberts, O. J., Fitzpatrick, G., \& Veres, P. 2016, GCN, 19411

Sari, R., \& Esin, A. A. 2001, ApJ, 548, 787

Sari, R., \& Piran, T. 1999, ApJ, 520, 641

Sari, R., Piran, T., \& Halpern, J. P. 1999, ApJL, 519, L17

Sari, R., Piran, T., \& Narayan, R. 1998, ApJL, 497, L17

Schulze, S., Klose, S., Bjornsson, G., et al. 2011, A\&A, 526, A23

Stratta, G., Fiore, F., Antonelli, L. A., Piro, L., \& De Pasquale, M. 2004, ApJ, 608,846

Tanvir, N. R., Levan, A. J., Cenko, S. B., et al. 2016, GCN, 19419

Welty, D. E., Xue, R., \& Wong, T. 2012, ApJ, 745, 173

Willingale, R., Starling, R. L. C., Beardmore, A. P., Tanvir, N. R., \& O'Brien, P. T. 2013, MNRAS, 431, 394

Woods, E., \& Loeb, A. 1995, ApJ, 453, 583

Yost, S. A., Harrison, F. A., Sari, R., \& Frail, D. A. 2003, ApJ, 597, 459

Zafar, T., Watson, D. J., Malesani, D., et al. 2010, A\&A, 515, A94

Zauderer, B. A., Berger, E., Margutti, R., et al. 2013, ApJ, 767, 161 